\title{
A Description of Discrete Internal Representation Schemes for Visual Pattern Discrimination
}

\author{
David H. Foster \\ Department of Communication and Neuroscience, University of Keele, Keele, Staffordshire, England
}

\begin{abstract}
A general description of a class of schemes for pattern vision is outlined in which the visual system is assumed to form a discrete internal representation of the stimulus. These representations are discrete in that they are considered to comprise finite combinations of "components" which are selected from a fixed and finite repertoire, and which designate certain simple pattern properties or features. In the proposed description it is supposed that the construction of an internal representation is a probabilistic process. A relationship is then formulated associating the probability density functions governing this construction and performance in visually discriminating patterns when differences in pattern shape are small. Some questions related to the application of this relationship to the experimental investigation of discrete internal representations are briefly discussed.
\end{abstract}

\section{Introduction}

Many of the contemporary schemes developed for the modelling of visual pattern recognition and discrimination involve the notion that the visual system represents stimuli internally as combinations of various discrete "components" which are drawn from some fixed repertoire, and which designate certain simple pattern properties. Visual recognition or discrimination of patterns is then supposed to be determined by the extent to which these pattern representations concur or differ (see Foster, 1977). Typical components suggested for these representations specify (i) global pattern features like symmetry, area, "compactness", and "jaggedness" (Sutherland, 1968; Mavrides and Brown, 1969; Aiken and Brown, 1971; see also Pitts and McCulloch, 1947), (ii) local pattern features like oriented lines, curves, and vertices (Gibson, 1969; Olson and Attneave, 1970; Beck, 1972; Beck and
Ambler, 1973; Rumelhart and Siple, 1974; Pomerantz, 1978), and (iii) spatial relations between local pattern features, such as "above", "right of", and "joined to" (Barlow et al., 1972; Sutherland, 1973; Reed, 1974; Palmer, 1978; Foster and Mason, 1979). For further examples and discussion, see Zusne (1970), Reed (1973), and Leeuwenberg and Buffart (1978).

Such discrete representation schemes may be contrasted with those schemes in which patterns are assumed to be represented internally by the visual system as unstructured, approximately point-for-point "images" of the stimulus. These pointillistic representations may be internally compared by applying to them certain smooth families of internal spatial transformations which act to bring the transformed representations as closely as possible into coincidence; recognition or discrimination of patterns is then supposed to be determined by the extent of the overlap or mismatch of the transformed representations (Pitts and McCulloch, 1947; Hoffman, 1970; Marko, 1973; Cooper and Shepard, 1978; Foster and Mason, 1979).

The purpose of this study is to outline a description of discrete pattern representation schemes and to formulate a relationship between performance in discriminating patterns and the construction by the visual system of the hypothesized discrete internal representations. In developing this relationship, a general approach will be adopted in which it will be explicitly assumed that the construction of an internal representation constitutes a probabilistic process. In a separate article (Foster, 1980), a technique for the experimental investigation of these discrete internal representations is proposed.

\section{Discrete Pattem Representations}

A pattern is considered here as any static 2- or 3 -dimensional distribution of light in the visual field. In attempting to formalize a general description of 
discrete internal representations of patterns, we shall find it convenient to distinguish two types of component in the representation. The distinction is modelled on the difference that may be considered to exist between the name of an object and what may be said about the object, the predicate. In this general description, it is supposed that there is a substrate for each internal representation which is drawn from a fixed and finite set $X$ of basis elements. At a primitive level, suitable basis elements might denote points, lines, and regions in the pattern. We shall be typically concerned with representations formed at this level, but the proposed description is also applicable at higher levels of representation where basis elements might consist of structured collections of primitive entities, these collections corresponding for example to the complex objects of a natural scene. The finite set $X$ of basis elements should therefore be interpreted as being context dependent. For each finite subset $\mathbf{x}=\left\{x_{1}, x_{2}, \ldots, x_{\xi}\right\}$ of basis elements $x_{\eta} \in X$, $\eta=1,2, \ldots, \xi$, incorporated in an internal representation there is considered to be associated with $\mathbf{x}$ a finite set $\alpha(\mathbf{x})=\left\{\alpha_{1}, \alpha_{2}, \ldots, \alpha_{\kappa}\right\}$ of variables ( $\kappa$, which depends on $\mathbf{x}$, may be unity) each of which defines some attribute appropriate to the entities which are denoted by the subset $\mathbf{x}$. These attributes $\alpha_{\mu}$, $\mu=1,2, \ldots, \kappa$, are supposed to be selected from a fixed and finite set $A$ of possible attributes, where $A$ depends on $X$. For example, if there is only one basis element in $\mathbf{x}$, that is, $\xi=1$, then, if that basis element denotes a point in the visual field, an appropriate attribute for $\mathbf{x}$ might designate some position of the point; if the basis element denotes a line, an appropriate attribute might designate some length of the line; and if the basis element denotes a region, an appropriate attribute might designate some area of the region.

As another illustration, when $\xi=2$, if the two basis elements $x_{1}, x_{2}$ in the subset $\mathbf{x}$ denote two distinct lines in the visual field, an appropriate attribute for $\mathbf{x}$ might designate some angle between the lines; if $x_{1}$ denotes a point and $x_{2}$ a non-intersecting nonhorizontal line, an appropriate attribute might designate their horizontal spatial ordering, that is, whether the point is left or right of the line; and if $x_{1}$ denotes a point and $x_{2}$ a region, an appropriate attribute might designate inclusion, that is, whether the point is inside or outside the region.

As a third illustration, when $\xi=3$, if the three basis elements $x_{1}, x_{2}, x_{3}$ in $\mathbf{x}$ denote three distinct points in the visual field, then an appropriate attribute might designate their alignment, that is, whether the points are collinear or noncollinear.

Note that, as in studies of machine-based pattern recognition (see, for example, Narasimhan, 1969; Rosenfeld, 1969; Fu, 1974), we distinguish between an. attribute and the values it may take. In machine-based pattern recognition, a distinction is sometimes also made between the cases here corresponding to $\mathbf{x}=\left\{x_{1}\right\}$ and $\mathbf{x}=\left\{x_{1}, x_{2}, \ldots, x_{\xi}\right\}, \xi \geqq 2$; for $\mathbf{x}=\left\{x_{1}\right\}$, the attributes associated with $\mathbf{x}$ are called properties and for $\mathbf{x}=\left\{x_{1}, x_{2}, \ldots, x_{\xi}\right\}, \xi \leqq 2$, some attributes associated with $\mathbf{x}$ may be interpreted as relations amongst the constituent $x_{\eta}, \eta=1,2, \ldots, \xi$ (Rosenfeld, 1969). For our purposes, it is convenient not to make this distinction.

In all of the above illustrations, the attributes are seen to be either continuous variables, for example the attribute designating the length of a line can assume any value in the interval $(0, \infty)$, or discrete variables, for example the attribute designating the relationship of a point to a region can assume only one of two values. In general, we shall suppose that the set $A$ of attributes may be partitioned into two subsets $A^{D}$ and $A^{C}$ : each attribute in $A^{D}$ can only assume values in a countable set which is equivalent to $\{1,2, \ldots, M\} \subset \mathbf{N}$, where $\mathbf{N}$ is the set of positive integers and $M$ depends on the attribute; and each attribute in $A^{C}$ can only assume values in some $l$-dimensional interval $\mathbf{I}$ in Euclidean space $\mathbf{R}^{l}$, where $\mathbf{R}$ is the set of real numbers, and $I$ and $l$ also depend on the attribute. In both cases, these attribute values are merely nominal; for example, the relations "left of" and "right of" might be labelled " 1 " and "2" respectively. Attributes in $A^{D}$ will be called discrete and those in $A^{C}$ will be called continuous. Typically, $M=2$, or 3 , and $l=1,2$, or 3 (Foster, 1980).

Other kinds of attribute may be defined; for instance, attributes might designate functions that specify the spatial power spectrum of the stimulus (see Rosenfeld, 1969; Ullmann, 1974). Only discrete and continuous attributes will be considered here.

Together, the finite set $X$ of basis elements and the finite set $A$ of attributes constitute the fixed and finite repertoire of components from which the internal representation is considered to be constructed.

A discrete internal representation is thus conceived of as comprising a finite set of finite subsets of basis elements, each subset in association with a finite number of attributes and attribute values. For example, a pattern consisting of a point and a line might be given the internal representation consisting of two basis elements $\left\{x_{1}, x_{2}\right\}$, the one labelled "point" and the other "line", an attribute $\alpha$ associated with $\left\{x_{1}, x_{2}\right\}$ which designates the perpendicular distance of the point from the line, and some value $a$ of that distance. Symbolically, the internal representation of a pattern may be generally written thus:

$$
\left\{\left(\mathbf{x}_{i} ;\left(\alpha_{i 1}, a_{i 1}\right),\left(\alpha_{i 2}, a_{i 2}\right), \ldots,\left(\alpha_{i \mathbf{x}(i)}, a_{i \kappa(i)}\right)\right): i=1,2, \ldots, n\right\}
$$

where, for $i=1,2, \ldots, n$, 
(i) basis-element subsets

$\mathbf{x}_{i}=\left\{x_{i 1}, x_{i 2}, \ldots, x_{i \xi(i)}\right\}, \quad x_{i \eta} \in X, \quad \eta=1,2, \ldots, \xi(i)$,

(ii) attributes

$\alpha_{i \mu} \in \boldsymbol{\alpha}_{i}\left(\mathbf{x}_{i}\right), \quad \mu=1,2, \ldots, \kappa(i)$,

(iii) attribute values

$a_{i \mu} \in\left\{\begin{array}{lll}\{1,2, \ldots, M\}, & \text { if } & \alpha_{i \mu} \in A^{D} \\ \mathbf{I} \subset \mathbf{R}^{l}, & \text { if } & \alpha_{i \mu} \in A^{C}\end{array}\right\}, \mu=1,2, \ldots, \kappa(i)$.

The quantities $M, \mathbf{I}$, and $l$ depend on $i$ and $\mu$. Note that this expression displays attributes and their values explicitly. The absence of any dependence of one attribute value on another; dependence that might occur in a hierarchical arrangement of attributes, is important for the method of analysis suggested here and developed in Foster (1980). The present approach does not preclude the analysis of attributes associated with complex basis elements which are themselves composed of more primitive basis elements, attributes and values. It does, however, require that the level of complexity for the description be defined appropriately in terms of the sets $X$ and $A$.

\section{Probabilistic Assignments}

It was said in the Introduction that the construction of an internal representation would be assumed to constitute a probabilistic process. The justification for this assumption is that there are a number of variable factors associated with the visual processing of pattern stimuli, for example the conditions of viewing and the observer's attentional state, the effect of which on the formation of an internal representation cannot be completely specified. This uncertainty gives rise to an effective indeterminism in the process. Also, there are some pattern stimuli which are inherently ambiguous, so that there are two or more equally appropriate internal representations that may be produced, one at a time (see Caglioti and Caianiello, 1978). Examples of such stimuli at a non-primitive level of pattern representation are the classical ambiguous figures, like the Necker cube and the Rubin's vase-faces illusion, the ambiguous forms generated by Shepard and Cermak (1973), and the ambiguous characters constructed by Blesser et al. (1973) and Naus and Shillman (1976). At a more primitive level of pattern representation, there are the ambiguous directional grouping effects obtained with arrays of simple figures described by Attneave (1968).

To take into account this proposed indeterminism in the formation of an internal representation, we suppose that the selection of basis-element subsets, the selection of attributes, and the selection of attribute values are each probabilistic processes. In what follows, we shall be particularly concerned with the identity of the attributes and the characteristics of the probability density functions governing the assignment of values to these attributes. The motivation for this approach will become apparent later, but it may be noted that it need not exclude the analysis of the probability density functions governing the selection of basis-element subsets. Any such probability density function may be analyzed in terms of an equivalent probability density function of a suitable discrete attribute associated with a new basis-element subset, as follows. Suppose an internal representation were to have a single basis-element subset chosen from the set $\left\{\mathbf{x}_{1}, \mathbf{x}_{2}, \ldots, \mathbf{x}_{n}\right\}$ of distinct subsets of basis elements, with probabilities $p(1), p(2), \ldots, p(n)$ respectively. Then, fix a "composite" basis-element subset $\mathbf{x}_{0}=\left\{\mathbf{x}_{1}, \mathbf{x}_{2}, \ldots, \mathbf{x}_{n}\right\}$ for the representation and associate with it a discrete attribute $\delta_{0}$ with values in $\{1,2, \ldots, n\}$ such that the probability of $\delta_{0}$ assuming the value $i$ is $p(i), i=1,2, \ldots, n$. To illustrate this notion, consider the probabilities of selection of one of two basis-element subsets labelled "spot" and "line". We could define a single basis-element subset labelled "figure" and a discrete attribute called "shape", and then consider the probabilities of the values "spot-like" and "line-like" being assigned to that attribute. An analogous procedure may be adopted for the analysis of the probability density functions governing the selection of attributes.

As was mentioned above, our interest here is in the identity of the attributes and the characteristics of the probability density functions associated with them. To establish the notation, let $\mathbf{x}_{i}$ and $\mathbf{x}_{j}$ be two arbitrary (and not necessarily distinct) subsets of basis elements selected for an internal representation, and suppose that a discrete attribute $\delta_{i \mu}$ is associated with $\mathbf{x}_{i}$ and that a continuous attribute $\gamma_{j v}$ is associated with $\mathbf{x}_{j}$. By definition, $\delta_{i \mu}$ is a discrete random variable, and $\gamma_{j v}$ is a continuous random variable. Let $p_{i \mu}$ be the conditional discrete probability density function of $\delta_{i \mu}$ and let $f_{j v}$ be the conditional continuous probability density function of $\gamma_{j v}$. Thus, $p_{t \mu}(m)$ is the probability that value $m \in\{1,2, \ldots, M\}$ is assigned to $\delta_{i \mu}$, and, for some subset $U$ in $\mathbf{R}^{l}, \int_{U} f_{f_{v}}(u) d u$ is the probability that the value of $\gamma_{j v}$ lies in $U$. In Foster (1980), some specific assumptions are made about the way that the shape of the density functions $p_{i \mu}$ and $f_{j v}$ might vary with changes in the pattern stimuli. Now we suppose only that, wherever they are defined, $p_{i \mu}$ and $f_{j v}$ vary smoothly with pattern shape. Since it is certain that on each occasion one (and only one) value is assigned to $\delta_{i \mu}$ and to $\gamma_{j w}$, it follows that $\sum_{m=1}^{M} p_{i \mu}(m)=1$ and $\int_{\mathbf{R}^{i}} f_{j v}(u) d u=1$, 
where $M$ depends on $i, \mu$, and $l$ depends on $j, v$. In the next section, we shall discuss bow these particular density functions may be related to performance in visual pattern discrimination.

\section{Discrimination of Patterns}

Let $B_{1}$ and $B_{2}$ be patterns. On each presentation to the visual system, each pattern is assumed to give rise to an internal representation consisting of a finite number of basis-element subsets, attributes, and attribute values, all selected probabilistically. We shall suppose that if the differences in shape between $B_{1}$ and $B_{2}$ are sufficiently small, the probabilities governing the selection for an internal representation of the basis-element subsets $\mathbf{x}_{i}$ and $\mathbf{x}_{r}$ and discrete and continuous attributes $\delta_{i \mu}$ and $\gamma_{j v}$ respectively are identical for $B_{1}$ and $B_{2}$; only the probability density functions $p_{i \mu}$ and $f_{j v}$ will be assumed to differ. (The parameters $i, j, \mu, v$ vary over finite ranges, and $i, j$ need not be distinct.)

To derive a relationship between these probability density functions and performance in discriminating the patterns $B_{1}$ and $B_{2}$, we return briefly to a consideration of the overall probabilities governing the assignment of internal representations to these patterns. We make the general assumption that pattern discriminability is determined by the differences in the probabilities of each of the internal representations which may be assigned to the patterns. The plausibility of this is seen from the following. Suppose that $B_{1}$ and $B_{2}$ each give rise to one of just two distinct internal representations $r_{1}$ and $r_{2}$. Let the probability that $B_{v}$ gives rise to $r_{w}$ be $p(v, w), v=1,2, w=1,2$. Hence,

$p(1,1)+p(1,2)=p(2,1)+p(2,2)=1$.

In the Introduction, it was mentioned that in discreterepresentation schemes the discriminability of patterns is supposed to be determined by the extent to which their internal representations differ. In the present condition, in which there are only two possible conjunctions of the internal representations for the patterns, namely $r_{1}$ and $r_{1}$ (or equivalently $r_{2}$ and $r_{2}$ ), and $r_{1}$ and $r_{2}$ (or equivalently $r_{2}$ and $r_{1}$ ), there are two limiting cases. First, suppose that $p(1,1)$ and $p(2,2)$ are each close to unity. $B_{1}$ and $B_{2}$ then give rise most of the time to different internal representations, and, on average, discriminability of $B_{1}$ and $B_{2}$ should be relatively high. From $(1), p(1,2)$ and $p(2,1)$ are each close to zero, whence the differences $|p(1,1)-p(2,1)|$ and $|p(1,2)-p(2,2)|$ are large. Second, suppose instead that $p(1,1)$ and $p(2,1)$ are each close to unity. $B_{1}$ and $B_{2}$ then give rise most of the time to the same internal representation, namely $r_{1}$, and, on average, discriminability should be relatively low. From $(1), p(1,2)$ and $p(2,2)$ are each close to zero, whence the differences $|p(1,1)-p(2,1)|$ and $|p(1,2)-p(2,2)|$ are small. Note that we are effectively translating a rule for discrimination based on differences in deterministic internal representations into one based on differences in assignment probabilities of non-deterministic internal representations. Note further that for this task of discriminating patterns that differ by small changes in shape, our concern is not with the nature of the difference between $r_{1}$ and $r_{2}$, but merely with the fact that there is a difference.

For the situation described at the beginning of this section, the consideration of these differences in probabilities reduces to the consideration of the differences in the probability density functions $p_{i \mu}$ and in the probability density functions $f_{j v}$ governing the assignment of discrete and continuous attribute values respectively. Let $p_{l \mu}(v, m), m \in\{1,2, \ldots, M\}$, and $f_{j v}(v, u)$, $u \in \mathbf{R}^{l}$, denote the different density functions for the patterns $B_{v}, v=1,2$. We also use the notation $p_{i \mu}(v, \cdot)$ and $f_{j v}(v, \cdot)$ for these functions. Observe that for each $v=1,2$ the function $p_{i \mu}(v, \cdot)$ is a sequence $\left(p_{i \mu}(v, 1), p_{i \mu}(v, 2), \ldots, p_{i \mu}(v, M)\right)$ with values in $\mathbf{R}$, and that the function $f_{j v}(v, \cdot)$ is a mapping of $\mathbf{R}^{l}$ into $\mathbf{R}$. An obvious way to measure the differences $\mid p_{i \mu}(1, m)$ $-p_{i u}(2, m) \mid, m \in\{1,2, \ldots, M\}$, and $\left|f_{j v}(1, u)-f_{j v}(2, u)\right|$, $u \in \mathbf{R}^{l}$, is with the $l_{p}$ and $L_{p}$ norms. Let

$e_{i \mu}(1,2)=\left(\sum_{m=1}^{M}\left|p_{t \mu}(1, m)-p_{l \mu}(2, m)\right|^{q}\right)^{1 / q}$,

$e_{j v}(1,2)=\left(\int_{\mathbf{R}^{l}}\left|f_{j v}(1, u)-f_{j v}(2, u)\right|^{q^{\prime}} d u\right)^{1 / q^{\prime}}$,

where $M, l$, and the integers $q, q^{\prime}\left(1 \leqq q, q^{\prime}<\infty\right)$ depend on $i, \mu, j, v$.

Since the subsets $\mathbf{x}_{i}, \mathbf{x}_{j}$ of basis elements, and the attributes $\delta_{i \mu}, \gamma_{j v}$ are each selected probabilistically, the effectiveness of the difference $e_{i \mu}(1,2)$ between $p_{i \mu}(1, \cdot)$ and $p_{i \mu}(2, \cdot)$ and the difference $e_{j v}(1,2)$ between $f_{j v}(1, \cdot)$ and $f_{j v}(2, \cdot)$ in determining the discriminability of $B_{1}$ and $B_{2}$ will depend on the values of these selection probabilities (which, by assumption, are the same for the two patterns). We accordingly suppose that $e_{i \mu}(1,2)$ and $e_{j v}(1,2)$ are weighted by coefficients $w_{l u}$ and $w_{j v}$ respectively, $0 \leqq w_{l \mu}, w_{j v} \leqq 1$, in their contribution to discriminability. To quantify this discriminability, we use the discrimination index $d^{\prime}$ which arises in the theory of signal detection (Green and Swets, 1966; Swets, 1973). (Although $d^{\prime}$ is here assumed to be based on normal-theory statistics, this does not imply that the continuous density functions $f_{j v}$ need be normal.) Recall that $d^{\prime}$ increases monotonically as the discriminability of the patterns increases, and $d^{\prime}=0$ corresponds to the non-discriminability of the patterns. Hence, each weighted difference term $w_{t \mu} e_{i \mu}(1,2)$, 
$w_{j v} e_{j v}(1,2)$ is supposed to contribute a factor $d_{i \mu}^{\prime}(1,2)$, $d_{j v}^{\prime}(1,2)$, depending monotonically on $w_{i \mu} e_{i \mu}(1,2)$, $w_{j v} e_{j v}(1,2)$ respectively, to the overall discriminability $d^{\prime}(1,2)$. Let these monotonic dependences be described by the functions $y_{i \mu}, y_{j v}$, thus:

$d_{i \mu}^{\prime}(1,2)=y_{i \mu}\left(w_{i \mu} e_{i \mu}(1,2)\right)$,

$d_{j v}^{\prime}(1,2)=y_{j v}\left(w_{j v} e_{j v}(1,2)\right)$.

Then,

$d^{\prime}(1,2)=\sum_{\text {all i } i \mu} d_{i \mu}^{\prime}(1,2)+\sum_{\text {all } j v} d_{j v}^{\prime}(1,2)$,

by the additivity of discrimination indices (see, for example, Durlach and Braida, 1969).

\section{Characteristics of Discrete and Continuous Attributes}

Equations (2)-(6) of Sect. 4 relate measurable pattern discrimination performance to the hypothesized probability density functions governing the assignment of attribute values. This relationship may be compared with the corresponding relationship which would be obtained in a scheme using unstructured pointillistic representations, as outlined in the Introduction. In such a scheme, we might suppose that a pattern is given an internal representation which has as basis elements some (non-countably) infinite number of internal "points" $x_{\zeta}$ parametrized by internal spatial coordinates $\zeta=\left(\zeta_{1}, \zeta_{2}\right) \in \mathbf{R}^{2}$. Associated with each $x_{\zeta}$ there is an attribute $g_{\zeta}$ which is a continuous random variable and which provides an estimate of the external coordinates $z^{\prime}=\left(z_{1}^{\prime}, z_{2}^{\prime}\right) \in \mathbf{R}^{2}$ of the external point denoted by $x_{\zeta}$. Let $g_{\zeta}$ be the continuous probability density function of $\varrho_{\xi}$. If the internal representation of the external point by $x_{\zeta}$ were, on average, veridical, then the expected value $E\left(Q_{r}\right)$ of $\varrho_{\zeta}$ would coincide with the actual coordinates $z$ of the external point, that is,

$E\left(\varrho_{\zeta}\right)=\int_{\mathbf{R}^{2}} z^{\prime} g_{\zeta}\left(z^{\prime}\right) d z^{\prime}=z$.

For the patterns $B_{1}$ and $B_{2}$, the differences $e_{\zeta}(1,2)$ in the probabilities governing the assignment of attribute values to $\varrho_{\zeta}$ for each internal point $x_{\zeta}$ are, by analogy with (3), thus:

$e_{\zeta}(1,2)=\left(\int_{\mathbf{R}^{2}}\left|g_{\zeta}\left(1, z^{\prime}\right)-g_{\zeta}\left(2, z^{\prime}\right)\right|^{q} d z^{\prime}\right)^{1 / q}$.

We might suppose that, as for discrete attributes, the contributions to pattern discriminability of these differences are weighted by factors $w_{\zeta}, 0 \leqq w_{\zeta} \leqq 1$, and that the relationship between the $w_{5},(1,2)$ and discriminability factors $d_{5}^{\prime}(1,2)$ is monotonic, thus:

$d_{\zeta}^{\prime}(1,2)=y_{\zeta}\left(w_{\zeta} e_{\zeta}(1,2)\right)$.
The overall discriminability of the patterns $B_{1}$ and $B_{2}$ is then given by

$d^{\prime}(1,2)=\int_{\text {al1 } \zeta} d_{\zeta}^{\prime}(1,2) d \zeta$,

where $d \zeta$ is the area element for the internal space $\mathbf{R}^{2}$ which provides the parameterization of the points $x_{\zeta}$.

In the particular case that $B_{2}$ differs from $B_{1}$ by a (small) displacement of a single point, the discriminability determined by (7)-(9) depends only on the magnitude and direction of the displacement. It does not depend on the spatial distribution of the points in $B_{1}$ that are not displaced. For the discrete scheme, the discriminability determined by (2)-(6) does not have this independence property.

The scheme summarized by (2) (6) is, however, characterized by a more distinctive property, namely one deriving from the difference term $e_{i \mu}(1,2)$ defined by (2) which directly embodies the discrete nature of the proposed representation. Suppose that we varied the shape of a pattern $B$ in some smooth fashion (formal definitions in Foster, 1980), where any particular shape is specified by a parameter $t$ ranging in some parameter space $T$ (a subspace of Euclidean space), that is, $B=B_{t}$ depends smoothly on $t$. Suppose we let $B_{1}=B_{t+\Delta t}$ and $B_{2}=B_{t-\Delta t}$ in (2)-(6), where $\Delta t>0$ is fixed and is in some sense small. Suppose further that the parameter space $T$ is chosen so that there are only a finite number of regions on which the objectively determined value of the discrete attribute $\delta_{l \mu}$ is constant. So far, no specific assumptions have been made about how the probability density functions $p_{i \mu}$ of the internally assigned values of $\delta_{i \mu}$ might depend on pattern shape. For the internally assigned values of $\delta_{i \mu}$ to coincide, on average, with the objectively determined values, the $p_{i \mu}$ might be constrained to have their greatest changes in value occur at or close to the boundaries between regions. If we could construct a curve $t(s), a \leqq s \leqq b$, in $T$ which has a finite number of intersections $s_{\theta}$ with these boundaries, then we should be able to observe peaks in the difference term $e_{i \mu}(1,2)$ and therefore in $d_{i \mu}^{\prime}(1,2)$ at or close to these critical values $s_{\theta}$ (Foster, 1980). [How the incremental differences in shape between $B_{1}$ and $B_{2}$ may be expressed in terms of the parameter $s$ is also discussed in Foster (1980).]

This form of variation with $t$ suggested for $e_{i \mu}(1,2)$ may be contrasted with that which might be expected for $e_{j v}(1,2)$. With no great loss in generality, suppose that the curve $t(s), a \leqq s \leqq b$, may be chosen so that the objectively determined value of the attribute $\gamma_{j v}$ varies linearly with $s$. A reasonable constraint on the distribution of the internally determined values of $\gamma_{j v}$ is that the mean or expected value $E\left(\gamma_{j v}\right)$ of $\gamma_{j v}$ should coincide with the objectively determined value, or at least with 
some value close to that. We might then further suppose that the parameterization of the curve $t(s)$, $a \leqq s \leqq b$, can be adjusted so that the second and higher moments about the mean of the distribution of $\gamma_{t v}$ are constant with $s$. Unlike the difference term $e_{i \mu}(1,2)$, the difference term $e_{j v}(1,2)$ should then be constant with $s$. If this were so, then $d_{j v}^{\prime}(1,2)$ would be constant with $s$.

Some implications of this claim are discussed in the next section.

\section{Discussion}

In this study, we have considered a class of schemes for visual pattern recognition and discrimination in which internal representations are assumed to be composed of combinations of components drawn from fixed and finite repertoires. For such discrete schemes, we have set out a description of these internal representations, taking into account their proposed probabilistic construction, and have formulated a relationship between observable pattern discrimination performance and the probability density functions governing the composition of the internal representations.

The intention in deriving this discrimination relationship has been to model the detectability of small changes in pattern shape. It has been assumed that each stimulus pattern is presented under conditions for which it is reasonable to suppose that just one internal representation is generated during that presentation. Scrutiny of the stimuli is assumed not to occur. The discrimination relationship is moreover not intended to cover the more complex kinds of process involved in visually comparing transformed (for instance, rotated and reflected) pairs of patterns, interpreted as "same", against randomly paired patterns, interpreted as "different", which have been analyzed by Foster (1978), Foster and Mason (1979), and Kahn and Foster (1980). The only transformation that here relates pairs of patterns assumed to give rise to identical probability density functions governing possible internal representations are the trivial translations in the plane perpendicular to the line of gaze. That is, internal representations differing only in their specification of pattern position are considered to be identical with respect to the discrimination relationship (but see Kahn and Foster, 1980).

The essential property of discrete representation schemes proposed here concerns the existence of discrete attributes. As indicated in Sect. 5, it should be possible to reveal the characteristics of the discrete probability density functions of these attributes by an examination of discrimination performance at points along suitable curves defining smooth variations in pattern shape. A number of difficulties have to be circumvented, however, before such a technique may be applied experimentally to the analysis of discrete internal representations. First, it is necessary to devise an adequate method for generating a smoothly varying family of pattern shapes. Unlike a formally analogous situation in auditory perception, where the auditory discriminability of adjacent speech-like sounds measured at points along an acoustic continuum has been found to maximize at the boundary of phonemes (Liberman et al., 1957, 1961), there are no natural "dimensions" to visual stimuli which can provide a suitable parameterization. Shepard and Cermak (1973) have devised a set of closed-curve visual stimuli specified by a finite-dimensional parameter space, and these have been used (Cermak, 1977) to explore a boundary effect in the "interpretation" of these stimuli, but such an approach is not sufficiently general for the present purpose.

Second, given that both discrete and continuous attributes may contribute towards discrimination performance, there is the problem of distinguishing these contributions. [Compare a similar problem in auditory perception; Pisoni and Lazarus (1974), see also Macmillan et al. (1977).] In principle, one would choose a parameterization of the patterns so that the contribution of all continuous attributes is constant with that parameter, as was suggested in Sect. 5. In practice, the selection of such a parameterization may not be obvious or straightforward.

Third, even with a suitable parameterization, the significance of the contribution to discrimination performance by some hypothesized discrete attribute may be masked if the contribution by continuous attributes is sufficiently large. Thus, the stimulus configuration and method of pattern presentation should somehow be chosen to preferentially weight the role of the discrete attributes in the internal representation.

An approach to the solution of these problems is proposed in Foster (1980). In that study two experiments are considered. The one examines the effect of a putative discrete attribute designating the collinearity or noncollinearity of the points in a pattern; the other tests for the existence of a discrete attribute designating the acuteness or obtuseness of the angle between two lines.

Acknowledgements. I thank D. M. MacKay, S. Gravano, J. I. Kahn, D. Lindsay, and R. J. Mason for many useful discussions in the preparation of this work.

\section{References}

Aiken, L.S., Brown, D.R.: A feature utilization analysis of the perception of pattern class structure. Percept. Psychopbys. 9, $279-283(1971)$

Attneave, F.: Triangles as ambiguous figures. Am. J. Psychol. 81, $447-453(1968)$ 
Barlow, H.B., Narasimhan, R., Rosenfeld, A.: Visual pattern analysis in machines and animals. Science 177, 567-575 (1972)

Beck, J.: Similarity grouping and peripheral discriminability under uncertainty. Am. J. Psychol. 85, 1-19 (1972)

Beck, J., Ambler, B.: The effects of concentrated and distributed attention on peripheral acuity. Percept. Psychophys. 14, 225-230 (1973)

Blesser, B., Shillman, R., Cox, C., Kuklinski, T., Ventura, J., Eden, M.: Character recognition based on phenomenological attributes. Visible Language 7, 209-223 (1973)

Caglioti, G., Caianiello, E.R.: A model for non-resolvable ambiguities. Biol. Cybernetics 31, 205-208 (1978)

Cermak, G.W.: Performance in a delayed comparison discrimination task as a function of stimulus interpretation. Percept Psychophys. 21, 69-76 (1977)

Cooper, L.A., Shepard, R.N.: Transformations on representations of objects in space. In : Handbook of perception, Vol 8. Carterette, E.C., Friedman, M.P. (eds.), pp. 105-146. New York, San Francisco, London: Academic Press 1978

Durlach, N.I, Braida, L.D.: Intensity perception. I. Preliminary theory of intensity resolution. J. Acoust. Soc. Am. 46, 372-383 (1969)

Foster, D.H. : Visual pattern recognition by assignment of invariant features and feature-relations. Opt. Acta 24, 147-157 (1977)

Foster, D.H.: Visual comparison of random-dot patterns: evidence concerning a fixed visual association between features and feature-relations. Q. J. Exp. Psychol. 30, 637-654 (1978)

Foster, D.H. : Spatial perturbation technique for the investigation of discrete internal representations of visual patterns. Biol. Cybernetics 38, 159-169 (1980)

Foster, D.H., Mason, R.J.: Transformation and relational-structure schemes for visual pattern recognition: Two models tested experimentally with rotated random-dot patterns. Biol. Cybernetics 32, 85-93 (1979)

Fu, K.S.: Syntactic methods in pattern recognition. New York, London: Academic Press 1974

Gibson, E.J.: Principles of perceptual learning and development. New York: Appleton-Century-Crofts 1969

Green, D.M., Swets, J.A.: Signal detection theory and psychophysics. New York, London, Sydney: Wiley 1966

Hoffman, W.C.: Higher visual perception as prolongation of the basic Lie transformation group. Math. Biosci. 6, 437-471 (1970)

Kahn, J.I., Foster, D.H.: Visual comparison of transformed patterns as a function of their positional symmetry and separation in the field (in preparation) (1980)

Leeuwenberg, E.L.J., Buffart, H.F.J.M. (eds.): Formal theories of visual perception. Chichester, New York, Brisbane, Toronto: Wiley 1978

Liberman, A.M., Harris, K.S., Hoffman, H.S., Griffith, B.C.: The discrimination of speech sounds within and across phoneme boundaries. J. Exp. Psychol. 54, 358-368 (1957)

Liberman, A.M., Harris, K.S., Kinney, J.A., Lane, H. : The discrimination of relative onset-time of the components of certain speech and nonspeech patterns. J. Exp. Psychol. 61, 379-388 (1961)

Macmillan, N.A, Kaplan, H.L., Creelman, C.D.: The psychophysics of categorical perception. Psychol. Rev. 84, 452-471 (1977)
Marko, H.: Space distortion and decomposition theory. A new approach to pattern recognition by vision. Kybernetik 13, 132-143 (1973)

Mavrides, C.M., Brown, D.R. : Discrimination and reproduction of patterns: feature measures and constraint redundancy as predictors. Percept. Psychophys. 6, 276-280 (1969)

Narasimhan, R: On the description, generation, and recognition of classes of pictures. In: Automatic interpretation and classification of images. Grasselli, A. (ed.), pp. 1-42. New York, London: Academic Press 1969

Naus, MJ., Shillman, R.J.: Why a $Y$ is not a $V$ : a new look at the distinctive features of letters. J. Exp. Psychol.: Hum. Percept Perform. 2, 394-400 (1976)

Olson, R.K., Attneave, F.: What variables procedure similarity grouping? Am. J. Psychol. 83, 1-21 (1970)

Palmer, S.E.: Structural aspects of visual similarity. Mem. Cognit. 6, 91-97 (1978)

Pisoni, D.B., Lazarus, J.H. : Categorical and noncategorical modes of speech perception along the voicing continuum. J. Acoust. Soc. Am. 55, 328-333 (1974)

Pitts, W., McCulloch, W.S.: How we know universals. The perception of auditory and visual forms. Bull. Math. Biophys. 9, $127-147$ (1947)

Pomerantz, J.R.: Are complex visual features derived from simple ones? In: Formal theories of visual perception. Leeuwenberg, E.L.J., Buffart, H.F.J.M. (eds.), pp. 217-229. Chichester, New York, Brisbane, Toronto: Wiley 1978

Reed, S.K.: Psychological processes in pattern recognition. New York, London: Academic Press 1973

Reed, S.K. Structural descriptions and the limitations of visual images. Mem. Cognit. 2, 329-336 (1974)

Rosenfeld, A. Picture processing by computer. New York, London: Academic Press 1969

Rumelhart, D.E., Siple, P.: Process of recognizing tachistoscopically presented words. Psychol Rev. 81, 99-118 (1974)

Shepard, R.N., Cermak, G.W.: Perceptual-cognitive explorations of a toroidal set of free-form stimuli. Cog. Psychol. 4, 351-377 (1973)

Sutherland, N.S. : Outlines of a theory of visual pattern recognition in animals and man. Proc. Roy. Soc. London, Ser. B 171, 297-317 (1968)

Sutherland, N.S.: Object recognition. In: Handbook of perception, Vol. 3. Carterette, E.C., Friedman, M.P. (eds.), pp. 157-185. New York, London: Academic Press 1973

Swets, J.A.: The relative operating characteristic in psychology. Science 182, 990-1000 (1973)

Ullmann, J.R: A review of optical pattern recognition techniques. Opto-electronics 6, 319-332 (1974)

Zusne, L.: Visual perception of form. New York, London: Academic Press 1970

Received: March 6,1980

Dr. D. H. Foster

Department of Communication and Neuroscience

University of Keele

Keele, Staffordshire ST5 5BG

England

Note added in proof. In this paper a form of non-discrete internal representation was outlined in which patterns are assumed to be represented as unstructured pointillistic "images". Internal comparison of these representations is considered to be achieved by applying certain smooth compensatory families of internal spatial transformations to the representations. The particular notion of dynamically effecting a family of internal spatial transformations as described by Cooper and Shepard (1978) does not itself require that the internal representations being transformed be pointillistic and unstructured (R. N. Shepard, 1980, personal communication); such representations may indeed be highly structured. See Shepard, R. N.: Psychophysical complementarity. In: Perceptual organization. Kubovy, M., Pomerantz, J. R. (eds.); Hillsdale, N. J.: Earlbaum 1980, for further discussion of the dynamics of internal pattern transformation; see also Foster, D. H.: Visual apparent motion and the calculus of variations. In: Formal theories of visual perception. Leeuwenberg, E. L. J., Buffart, H. F. J. M. (eds.), pp. 67-82; Chichester: Wiley 1978. 